\section{Decolonization of multi-drug resistant bacteria by fecal microbiota transplantation in five pediatric patients before allogeneic hematopoietic stem cell transplantation: gut microbiota profiling, infectious and clinical outcomes}

Fecal microbiota transplantation (FMT) is playing a prominent role in the treatment of recurrent Clostridium difficile infection in adults, showing high efficacy and safety. ${ }^{1}$ It has also been proposed for treatment of other diseases associated with alterations of intestinal microbiota, including intestinal inflammatory diseases (inflammatory bowel disease) ${ }^{2}$ and graft-versus-host disease (GvHD). ${ }^{3}$ Moreover, FMT has been proposed for decolonization of multi-drug resistant (MDR) germs from the intestinal tract, with good results in adults. ${ }^{4,5}$ Indeed, antimicrobial resistance (AMR) is of great concern in the hemato-oncologic field, since a very high mortality rate has been demonstrated in patients infected by MDR bacteria (up to $36-95 \%$ in patients undergoing allogeneic hematopoietic stem cell transplantation [HSCT]). ${ }^{6}$ Moreover, up to $70 \%$ of cases of bacteremia originate from the gut in these patients.

Between October 2018 and March 2019, five consecutive patients colonized by MDR bacteria underwent FMT before HSCT (see Table 1 for details), on a compassionate use basis after local ethical committee approval and informed consent of parents/legal guardians of patients. Notably, three patients had a prior history of systemic infections by a colonizing MDR pathogen, which had required intensive care unit admission. Isolated MDR pathogens were Pseudomonas aeruginosa and carbapenemresistant Enterobacteriaceae (CRE) (Table 1); three patients had isolation of different species of CRE in stools (Figure 1). Real-time polymerase chain reaction (RT-PCR) targeting carbapenemases detected $b l a_{\mathrm{VIM}}$ in three cases, $b l a_{\mathrm{NDM}}$ in one case, and $b l a_{\mathrm{OXA} 48-181-232}$ in another case.

For each subject, the same unrelated healthy volunteer adult donor was used. Donor screening was performed according to European consensus guidelines on FMT, ${ }^{1}$ our institutional FMT protocol and Italian recommendations

Table 1. Characteristics of patients undergoing fecal microbiota transplantation (FMT) for multidrug resistant (MDR) decolonization.

\begin{tabular}{|c|c|c|c|c|c|}
\hline & Patient 1 & Patient 2 & Patient 3 & Patient 4 & Patient 5 \\
\hline Age (years) at FMT & 18 & 17 & 11 & 9 & 2 \\
\hline Gender & M & M & M & M & $\mathrm{F}$ \\
\hline Hematologic disease & AML & AML & AML & ALL & SCID \\
\hline Phase of disease & CR1 & CR1 & CR1 & CR2 & Disease present \\
\hline MDR pathogen & $\mathrm{PA}$ & CF, KOr, EntCl & $\mathrm{KP}, \mathrm{EC}$ & $\mathrm{EC}$ & $\mathrm{EC}, \mathrm{KO}, \mathrm{KP}$ \\
\hline AMR gene & $b l a_{\text {VM }}$ & $b l a_{\mathrm{VM}}$ & $b l a_{\mathrm{VM}}$ & $b l a_{0 \times A 48-181-232}$ & $b l a_{\mathrm{NDM}}$ \\
\hline Pre-FMT relevant infections & Sepsis by Carb-R-PA & Sepsis by Carb-R-EC & None & None & $\begin{array}{l}\text { Multiple sepsis and } \\
\text { meningoencephalitis } \\
\text { by Carb-R-EC }\end{array}$ \\
\hline Donor & UD & UD & UD & UD & UD \\
\hline Stool & Fresh & Frozen & Frozen & Frozen & Frozen \\
\hline N. of FMT & 1 & 1 & 1 & 1 & 1 \\
\hline Volume administered & $170 \mathrm{~mL}$ & $200 \mathrm{~mL}$ & $150 \mathrm{~mL}$ & $240 \mathrm{~mL}$ & $100 \mathrm{~mL}$ \\
\hline Preparation with oral antibiotic & $\mathrm{N}$ & Y & $\mathrm{Y}$ & $\mathrm{Y}$ & $\mathrm{Y}$ \\
\hline MDR bacteria clearance at 1 week & Y & Y & $\mathrm{N}$ & Y & Y \\
\hline MDR bacteria clearance at 1 month & $\mathrm{N}$ & $\mathrm{N}$ & Y & $\mathrm{N}$ & $\mathrm{N}$ \\
\hline MDR bacteria clearance at last follow-up & $\mathrm{N}$ & $\mathrm{N}$ & $\mathrm{Y}$ & $\mathrm{N}$ & $\mathrm{N}$ \\
\hline Last microbiological follow-up (days) & 44 & 28 & 53 & 42 & 113 \\
\hline Time elapsing between FMT and HSCT & 12 days & 14 days & 13 days & 16 days & 16 days \\
\hline Type of donor and stem cell source & $\begin{array}{l}\text { HLA-haploidentical } \\
\text { relative, PBSC }\end{array}$ & $\begin{array}{l}\text { HLA-haploidentical } \\
\text { relative, PBSC }\end{array}$ & $\begin{array}{l}\text { HLA-identical } \\
\text { sibling, BM }\end{array}$ & $\begin{array}{l}\text { HLA-haploidentical } \\
\text { relative, PBSC }\end{array}$ & $\begin{array}{l}\text { HLA-haploidentical } \\
\text { relative, PBSC }\end{array}$ \\
\hline Conditioning regimen & $\begin{array}{l}\text { TBI + TT + LPAM + } \\
\text { ATLG + rituximab }\end{array}$ & $\begin{array}{l}\text { TBI + TT + LPAM + } \\
\text { ATLG + rituximab }\end{array}$ & $\begin{array}{l}\mathrm{Bu}+\mathrm{Cy}+ \\
\mathrm{LPAM}\end{array}$ & $\begin{array}{l}\text { TBI + TT + } \\
\text { Flu + ATLG } \\
\text { + rituximab }\end{array}$ & $\begin{array}{l}\text { Treo + Flu + } \\
\text { ATLG + rituximab }\end{array}$ \\
\hline Graft manipulation & TCR $\alpha \beta / C D 19$-depletion & TCR $\alpha \beta / C D 19$-depletion & None & TCR $\alpha \beta / C D 19-d e p l e t i o n$ & TCR $\alpha \beta / C D 19-d e p l e t i o n$ \\
\hline CD34+ infused/kg & $9.6 \times 10^{6}$ & $14.4 \times 10^{6}$ & $5.2 \times 10^{6}$ & $18.2 \times 10^{6}$ & $18.0 \times 10^{6}$ \\
\hline Engraftment $\mathrm{Y} / \mathrm{N}$, days & $\mathrm{Y}, 9$ & $\mathrm{Y}, 12$ & $\mathrm{Y}, 17$ & $\mathrm{Y}, 16$ & $\mathrm{Y}, 12$ \\
\hline Acute/chronic GvHD & $\mathrm{N}$ & $\begin{array}{l}\text { Y, acute, grade I, } \\
+40 \text { after HSCT }\end{array}$ & $\mathrm{N}$ & $\mathrm{N}$ & $\mathrm{N}$ \\
\hline Follow-up (days after FMT) & 302 & 264 & 204 & 162 & 120 \\
\hline
\end{tabular}

AMR: antimicrobial resistant;ALL: acute lymphoblastic leukemia;AML: acute myeloid leukemia;ATLG: anti-T lymphocyte globulins; bla $_{\mathrm{NDM}}$ :New Delhi metallo-beta-lactamase; bla $_{\mathrm{VM}}$ : Verona imipenenase; bla $a_{\mathrm{OXA}}$ oxacillinase; BM: bone marrow; Bu: busulfan; CF: Citrobacter freundii; Carb-R: carbapenem-resistant; CR: complete remission; Cy: cyclophosphamide; EC: Escherichia coli; EntCl: Enterobacter cloacae; Flu: fludarabine; GvHD: graft-versus-host disease; HSCT: hematologic stem cell transplantation; KP: Klebsiella pneumoniae; KOr: Klebsiella ornithinolytica; KO: Klebsiella oxytoca; LPAM: melphalan; M: male; N: number; PA: Pseudomonas aeruginosa; PBSC: peripheral blood stem cells; SCID: severe combined immune deficiency; TBI: total body irradiation; TCR: T-cell receptor; Treo: treosulfan; TT: thiotepa; UD: universal donor. 
of the National Health Authority (Consiglio Superiore Sanità) (http://www.regione.lazio.it/binary/rl_sanita/tbl_normativa/SAN_DCA_U00111_14_03_2019.pdff). FMT emulsion was prepared under aerobic conditions, from either frozen or fresh preparation, according to stool bank availability and clinical need. FMT infusion was performed via esophagogastroduodenoscopy (EGDS) in the duodenum. A naso-gastric tube was placed after FMT in order to protect the patient from vomiting/inhalation. During the week before FMT, no systemic antibiotics were administered. All patients, except one, received a 3-day course of oral colistin before FMT to improve decolonization efficacy. Stool sample collection was performed at the FMT day (T0), day 1(T1), 3(T2), $7 \pm 1(\mathrm{~T} 3), 10 \pm 2(\mathrm{~T} 4)$, $20 \pm 3$ (T5), $25 \pm 2$ (T6), $28 \pm 2$ (T7), according to patients clinical condition and sample availability.

After a minimum of three days post-FMT, based on physician's evaluation, the conditioning regimen for HSCT was started. Anti-infectious prophylaxis/treatment strategy is reported in the Online Supplementary Appendix. Adverse events (AE) were graded according to Common Terminology Criteria for Adverse Events (CTCAE), version 4.03. MDR bacteria surveillance and gut microbiota profiling are reported in the Online Supplementary Appendix.

Multi-drug resistant decolonization was achieved within one week in 4 of 5 patients $(80 \%)$, for whom RTPCR for bla alleles resulted negative on stools. However, at 1-month follow-up, the only patient who was still colonized after FMT, achieved decolonization, while the four previously decolonized patients switched to a new colonization status (from the same pathogen identified before FMT) (see Figure 1 for details). At last microbio- logical follow-up (mean time 56 days, range 28-113 days), 4 of 5 patients were colonized by MDR pathogens. Details on gut microbiota ecology are reported in Figure 2 and in the Online Supplementary Appendix.

Patient 1, after achieving decolonization, experienced a sepsis due to the same MDR-colonizing pathogen (Figure 1) 5 days post-HSCT (17 days after FMT). Patient 5 experienced a sepsis 24 hours after FMT from the same pathogen colonizing her stools; however, after careful clinical revision, this was attributed to contamination of the central venous line by the caregiver. Both episodes were promptly treated with targeted antibiotics, and patients fully recovered without sequelae. The remaining three patients did not present any major infective episodes. Other AE recorded were nausea (two patients with grade 1 and 2, respectively), abdominal pain (grade 1 ), and bloating (grade 1) in single patients.

Globally, FMT for MDR decolonization in pediatric patients has been reported only twice. The first case refers to a 14-year-old patient treated for hemophagocytic lymphohistiocytosis, experiencing recurrent carbapenem-resistant Klebsiella pneumoniae (CR-KP) infections, successfully decolonized by FMT, and with no recurrence of infection in the following 1.5 years. ${ }^{8}$ The second patient described in a recent retrospective study focusing on adult hematologic patients was a 16-year-old female with acute myelogenous leukemia. ${ }^{9}$ This patient underwent two FMT for VRE (Vancomycin-resistant Enterococci) and CP-producing bacteria colonization 98 days after HSCT, resulting in decolonization of VRE and persistence of carbapenemase (CP)-producing bacteria, with no reported AE.

In our study, FMT using samples from the same donor

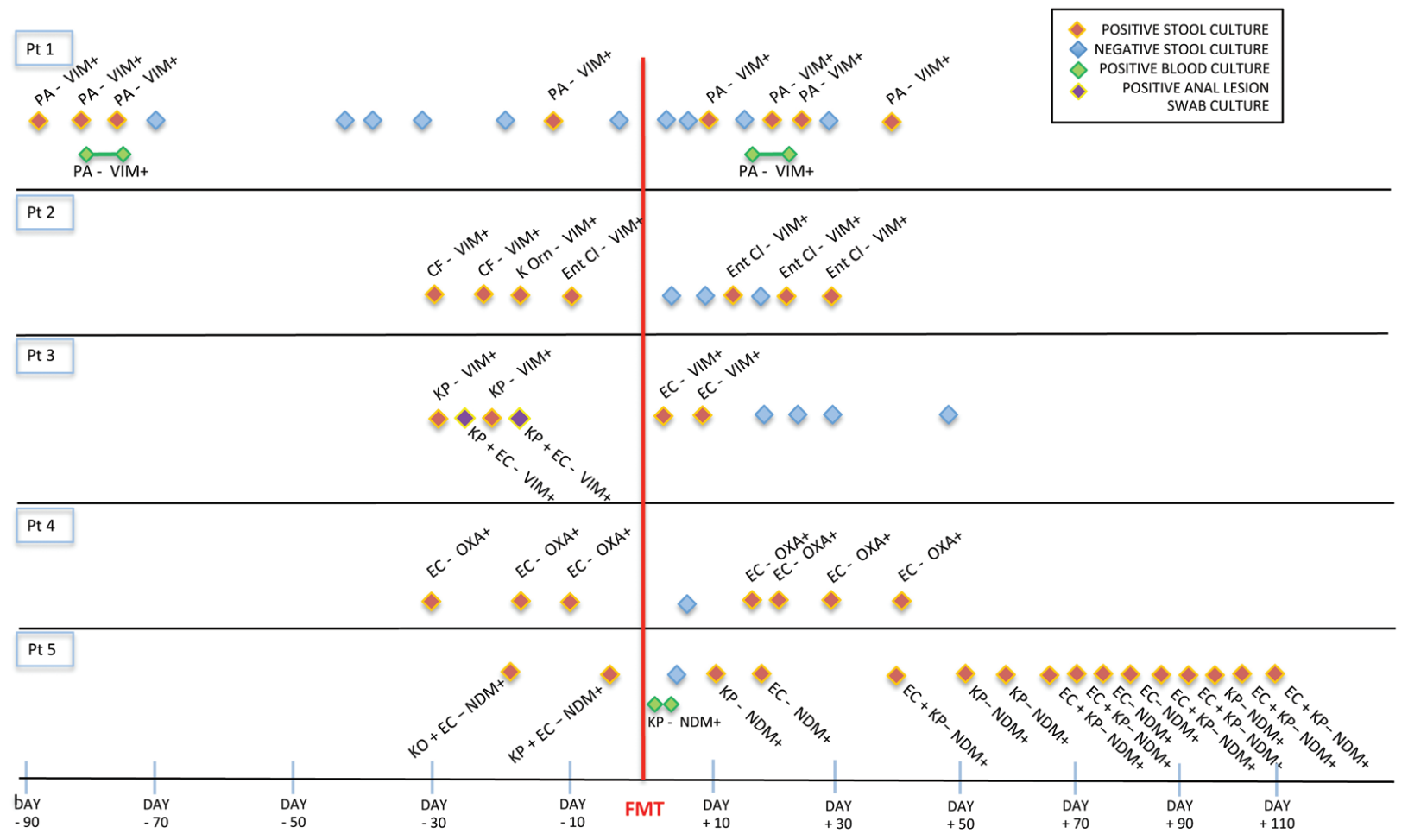

Figure 1. Distribution of multidrug resistant (MDR) pathogens isolated before and after fecal microbiota transplantation (FMT) and characterized. CF: Citrobacter freundii; EC: Escherichia coli; EntCl: Enterobacter cloacae; KP: Klebsiella pneumoniae; Kor: Klebsiella ornithinolytica; KO: Klebsiella oxytoca; NDM: New Delhi metallo-beta-lactamase; OXA: oxacillinase; PA: Pseudomonas aeruginosa; Pt: patient; VIM: Verona imipenenase. 


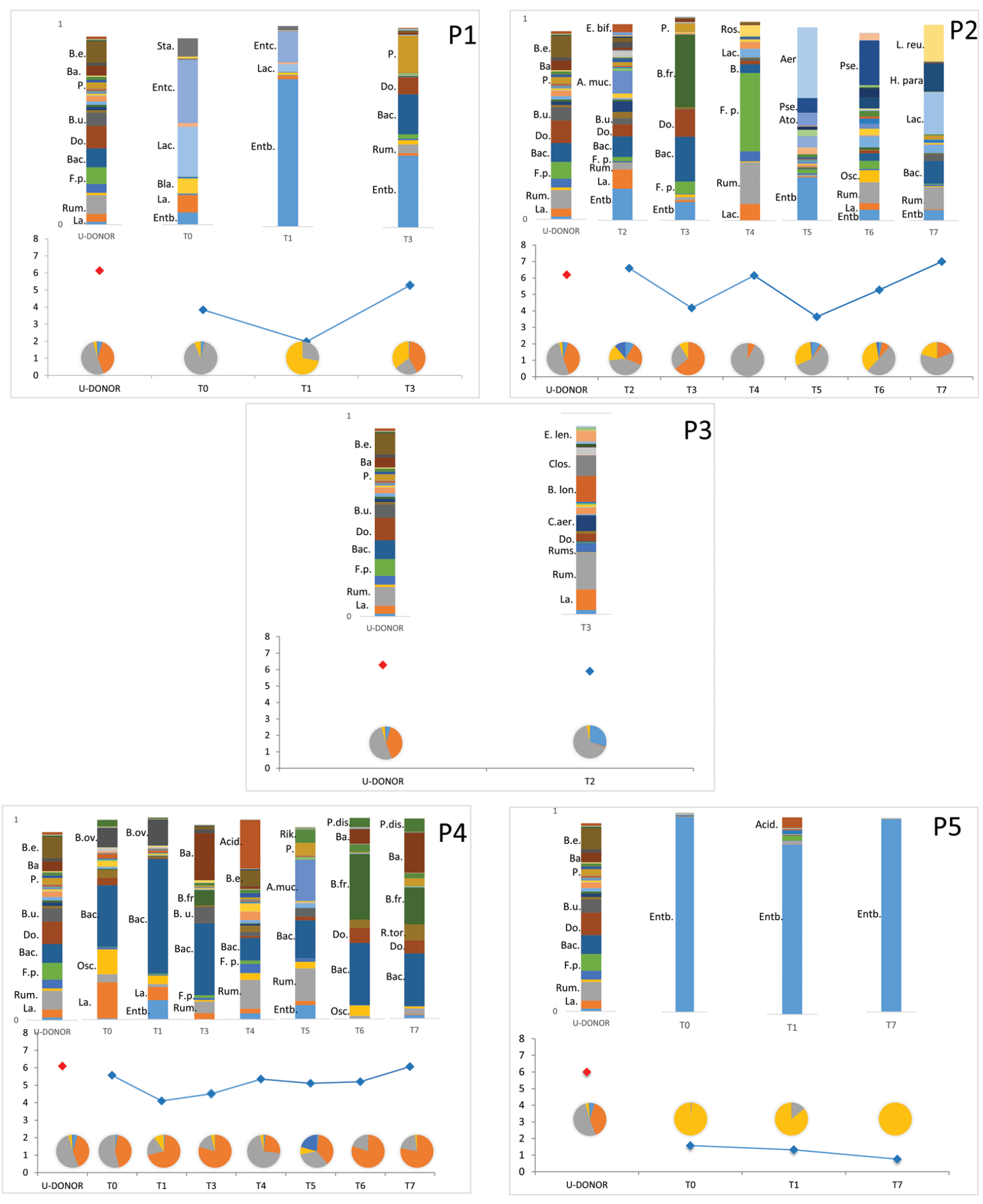

\section{Actinobacteria $\quad$ Bacteroidetes $\quad$ Firmicutes $\quad$ Proteobacteria $\quad$ Verrucomicrobia}

Figure 2. Targeted-metagenomics-based gut microbiota profiling. Barr plots represent operational taxonomic unit distribution of universal donor (U-DONOR) and patient follow-up time-points; line graph represents the Shannon index values for each time points; pie charts report phylum distributions for each time points. Histogram abbreviations: Acid.: Acidaminococcus; A.muc.: A. muciniphila; Aer.: Aerococcaceae; Ato.: Atopobium; B.e.: B. eggerthii; B.fr.: B. fragilis; B.Ion.: B. longum; B.ov.: B. ovatus; B.u.: B. uniformis; Bac.: Bacteroides; Ba.: Barnesiellaceae; Bla.: Blautia; C.aer.: C. aerofaciens; Clos.: Clostridiaceae; Do.: Dorea; E.len.: E. Ienta; E.bif.: E.biforme; Entb.: Enterobacteriaceae; Entc.: Enterococcaceae; F.p.: F. prausnitzii; H.para.: H. parainfluenzae; L.reu.: L. reuteri; La., Lachnospiraceae; Lt.: Lactobacillus; Osc., Oscillospira; P., Parabacteroides; P. dis.: P. distasonis; Pse.: Pseudomonas; Ros.: Roseburia; Rum.: Ruminococcaceae; Rums.: Ruminococcus; Rik.: Rikenellaceae; R.tor.: R. torques; Sta.: Staphylococcus. 
resulted in $80 \%$ (4 of 5) MDR decolonization within one week after the procedure. Since repeated FMT could increase the chances of durable decolonization, this protocol might be indicated for patients with a predicted long-lasting immunosuppression or in the presence of other risk factors for recurrent infections.

Our data suggest FMT safety and feasibility in pediatric patients with hematologic disorders immediately before the aplastic phase of HSCT. Four patients did not experience serious $\mathrm{AE}$, while patient 1 suffered from an episode of sepsis (from the same pathogen for which he received FMT) 17 days after the procedure. In all patients, only few symptoms related to the FMT procedure were recorded, all being transient and easily controlled by symptomatic drugs. Recently, two episodes of life-threatening/fatal sepsis due to ESBL E. coli were reported in adults undergoing FMT in two different clinical trials. ${ }^{10}$ However, based on the last European Consensus ${ }^{11}$ and national recommendations, extensive MDR bacteria testing is a cornerstone of donor screening in our institution.

Antibiotic-driven decolonization is a matter of discussion in the context of FMT. Oral colistin was proposed as a treatment to decolonize gut microbiota MDR bacteria before FMT. Stoma et al. randomized 62 adult hematologic patients colonized by MDR bacteria to receive oral colistin or placebo for 14 days, ${ }^{12}$ showing improved decolonization at the end of treatment not persisting 1 week later; moreover, the incidence of bloodstream infections in the case/control groups was similar. Based on these results, oral colistin was administered to four of our patients before FMT to provide an "induction" therapy followed by "consolidation" through FMT, with the idea of improving donor microbiota "engraftment". From our data, it seems that, at early time points after FMT preceded by oral colistin administration, the gut microbiota composition was more similar to that of the FMTdonor. However, more patients are needed to assess the effective role of oral colistin as FMT preparation on microbiome composition.

After the exclusion of related donors, who were not eligible because of the presence of pathogens/commensals at screening, a healthy unrelated volunteer was selected, in agreement with previous literature. Indeed, systematic reviews/meta-analysis ${ }^{13}$ in patients receiving FMT for $C$. difficile infection did not report any difference in outcomes based on donor selection. Donor (unrelatedversus-related) and sample (fresh-versus-frozen) types are emerging topics, since availability of stool banks could widen and facilitate FMT, ${ }^{14}$ especially under emergency regimes. We used both fresh and frozen emulsions from the Ospedale Pediatrico Bambino Gesù FMT bank. Since the screening of potential donors can require weeks, the use of frozen material can reduce the time to perform the procedure.

We administered the stool preparation during EGDS directly in the duodenum. Battipaglia et al. used enema as a way of administration, reporting good rate of decolonization. ${ }^{9}$ We preferred upper gastrointestinal tract (GI) administration to extend as much as possible the effect of FMT to the whole intestine. ${ }^{15}$ Current literature reports the administration via upper GI in most cases.

Results obtained in 4 of 5 patients suggest that microbiota changes following FMT occur after T1, when its composition is still similar to T0. In particular, the recipients' microbiota seems to be colonized by donor bacteria starting from one week after the procedure. These similarities are not long-lasting. Indeed, at approximately 10 days after FMT, the recipients' microbiota display a consistently different profile both from the donor and the recipient's T0. These changes could be secondary to the conditioning regimen and/or antibiotic prophylaxis. Therefore, boosts of FMT should be considered within one week after first procedure to consolidate MDR decolonization (e.g., leaving in place a naso-jejunale tube in pediatric patients).

From an ecological point of view, 1 day after FMT we recorded the overgrowth of facultative anaerobes and aerobes, as Enterobacteriaceae, probably promoted by the $\mathrm{O}_{2}$-conditions generated during the FMT emulsion. Afterward, the slow growth of strict anaerobes from donor reduced the $\mathrm{O}_{2}$-conditions suppressing the relative amount of Enterobacteriaceae. Thus, we suggest that future FMT emulsion preparations should be performed under anaerobic conditions to reduce the Enterobacteriaceae overgrowth favoring MDR species.

Main limitations of this report are: (i) its non-prospective nature; (ii) some heterogeneity of the FMT protocol (e.g., use of colistin, fresh/frozen material); (iii) the small number of cases, all affecting the possibility to draw firm conclusions.

In conclusion, we showed that FMT for MDR-decolonization of pediatric hematologic patients is safe, feasible, and effective in the short-term. Stool preparations from universal donors, starting from either fresh or frozen material, are readily available and safe, thus paving the way to stool banks. Further studies enrolling more populations are needed to confirm these preliminary data and to improve effectiveness of decolonization and infection clearance during the HSCT window.

Pietro Merli, ${ }^{1 *}$ Lorenza Putignani, ${ }^{2 *}$ Annalisa Ruggeri, ${ }^{1}$ Federica Del Chierico, ${ }^{3}$ Livia Gargiullo, ${ }^{4}$ Federica Galaverna, Stefania Gaspari, ${ }^{1}$ Daria Pagliara, ${ }^{1}$ Alessandra Russo, ${ }^{3}$ Stefania Pane, ${ }^{5}$ Luisa Strocchio, ${ }^{1}$ Mattia Algeri, Francesca Rea, ${ }^{6}$ Erminia Francesca Romeo, ${ }^{6}$ Paola Bernaschi, ${ }^{7}$ Andrea Onetti Muda, ${ }^{8}$ Bruno Dallapiccola ${ }^{9}$ and Franco Locatelli, ${ }^{i, 10}$

${ }^{1}$ Department of Hematology/Oncology, Cellular and Gene Therapy, Bambino Gesù Children's Hospital; '2Unit of Parasitology and Unit of Human Microbiome, Bambino Gesù Children's Hospital; ${ }^{3}$ Unit of Human Microbiome, Bambino Gesù Children's Hospital; ${ }^{4}$ Unit of Immunology and Infectious Diseases, University-Hospital Pediatric Department, Bambino Gesù Children's Hospital; 'Unit of Parasitology, Bambino Gesù Children's Hospital; 'Digestive Endoscopy and Surgery Unit, Bambino Gesù Children's Hospital; 'Unit of Microbiology, Bambino Gesù Children's Hospital; ${ }^{8}$ Department of Laboratories, Bambino Gesù Children's Hospital; 'Scientific Directorate, Bambino Gesù Children's Hospital and ${ }^{10}$ Sapienza, University of Rome, Rome, Italy

*PM and LP contributed equally as co-first authors.

Correspondence:

PIETRO MERLI - pietro.merli@opbg.net

doi:10.3324/haematol.2019.244210

Funding: this study was partially supported by the Italian Ministero della Salute (Bando Ricerca Finalizzata 2013, Giovani Ricercatori section, code GR-2013-02357136 to PM).

Acknowledgments: we wish to thank the FMT Committee of Bambino Gesì Children's Hospital and research Institute (Giulia Angelino, Marta Argentieri, Luigi Dall'Oglio, Patrizia D'Argenio, Paola De Angelis, Simona Faraci, Andrea Finocchi, Gianluca Foglietta, Sandra Martino, Giulia Marucci, Andrea Quagliariello, Giuliano Torre, Filippo Torroni, Valerio Nobili).

\section{References}

1. Cammarota G, Ianiro G, Tilg H, et al. European consensus conference on faecal microbiota transplantation in clinical practice. Gut. 
2017;66(4):569-580.

2. Fang H, Fu L, Wang J. Protocol for fecal microbiota transplantation in inflammatory bowel disease: a systematic review and meta-analysis. BioMed Res Int. 2018;2018:8941340.

3. Spindelboeck W, Schulz E, Uhl B, et al. Repeated fecal microbiota transplantations attenuate diarrhea and lead to sustained changes in the fecal microbiota in acute, refractory gastrointestinal graft-versushost-disease. Haematologica. 2017;102(5):e210-e213.

4. Bilinski J, Grzesiowski P, Sorensen N, et al. Fecal microbiota transplantation in patients with blood disorders inhibits gut colonization with antibiotic-resistant bacteria: results of a prospective, single-center study. Clin Infect Dis. 2017;65(3):364-370

5. Singh R, de Groot PF, Geerlings SE, et al. Fecal microbiota transplantation against intestinal colonization by extended spectrum beta-lactamase producing Enterobacteriaceae: a proof of principle study. BMC Res Notes. 2018;11(1):190

6. Caselli D, Cesaro S, Ziino O, et al. Multidrug resistant Pseudomonas aeruginosa infection in children undergoing chemotherapy and hematopoietic stem cell transplantation. Haematologica. 2010; 95(9):1612-1615.

7. Samet A, Sledzinska A, Krawczyk B, et al. Leukemia and risk of recurrent Escherichia coli bacteremia: genotyping implicates E. coli translocation from the colon to the bloodstream. Eur J Clin Microbiol Infect Dis. 2013;32(11):1393-1400.

8. Freedman A, Eppes S. Use of stool transplant to clear fecal coloniza- tion with carbapenem-resistant Enterobacteraciae (CRE): proof of concept. Open Forum Infect Dis. 2014;(Suppl 1):S65.

9. Battipaglia G, Malard F, Rubio MT, et al. Fecal microbiota transplantation before or after allogeneic hematopoietic transplantation in patients with hematologic malignancies carrying multidrug-resistance bacteria. Haematologica. 2019;104(8):1682-1688.

10. Defilipp Z, Bloom PP, Torres Soto M, et al. Drug-resistant E. coli bacteremia transmitted by fecal microbiota transplant. $N$ Engl J Med. 2019;381(21):2043-2050.

11. Cammarota G, Ianiro G, Kelly CR, et al. International consensus conference on stool banking for faecal microbiota transplantation in clinical practice. Gut. 2019;68(12):2111-2121.

12. Stoma I, Karpov I, Iskrov I, et al. Decolonization of intestinal carriage of MDR/XDR gram-negative bacteria with oral colistin in patients with hematological malignancies: results of a randomized controlled trial. Mediterr J Hematol Infect Dis. 2018;10(1):e2018030.

13. Li YT, Cai HF, Wang ZH, Xu J, Fang JY. Systematic review with metaanalysis: long-term outcomes of faecal microbiota transplantation for Clostridium difficile infection. Aliment Pharmacol Ther. 2016; 43(4):445-457.

14. Terveer EM, van Beurden YH, Goorhuis A, et al. How to: establish and run a stool bank. Clin Microbiol Infect. 2017;23(12):924-930.

15. Kamada N, Chen GY, Inohara N, Nunez G. Control of pathogens and pathobionts by the gut microbiota. Nat Immunol. 2013; 14(7):685-690. 